\title{
Exploring Cooperation in Traffic and Transportation Systems: Concepts, Principles and Approaches
}

\author{
Xingguang CHEN* \\ School of Business, Jianghan University, Wuhan City, \\ P.R.China 430056 \\ Yunhai DAI \\ School of Business and Management, Namseoul \\ University, Seoul, Korea 31020
}

\author{
Zhentao ZHU \\ College of Economics and Management, Nanjing \\ Institute of Technology, Nanjing City, P.R.China 211167
}

\begin{abstract}
Facilitating cooperation on all spatiotemporal and decision scales and creating cooperative transport systems are critical and urgent solutions for increasingly emerging urban traffic challenges. The purpose of this paper is to theoretically explore the general mechanism of cooperative formation and development and to propose an approach to cultivate and facilitate cooperation in urban traffic by leveraging big data. An conceptual framework named public information pools (PIP) to resolve collective irrationality accompanied by traveler selfish choices combined with comparison of some distinguished social dilemma solve approaches, for instance, Pigouivain theory, Coase theorem and social involvement model are presented.
\end{abstract}

Keywords-Urban traffic, Cooperation, Decision making, Big data, Game, Collective irrationality

\section{INTRODUCTION}

Traffic and transportation systems are facing unprecedented challenges for many years. Increasing travel demand combined with physical, social and environmental constraints are leading to strained and saturated traffic and transportation systems. In some developing countries, such as China, are experiencing rapid increase of motor vehicle number for the development of urbanization, industrialization and motorization. The overall Chinese motor vehicle ownership is more than 290 million including 194 million cars by the end of 2016 . There are 49 Chinese cities that have more than one million motor vehicles. Additionally, traffic emissions from transportation are unlikely to diminish while vehicle numbers continue to escalate in China's cities. Rapid motorization produces congestion, excessive injuries and fatalities, environment pollution and urban sprawl. All these lead to unsustainable transportation monoculture and inevitably, a rigorous challenge for sustainable development of China(Black 1996).

Urban traffic and transportation system belongs to complex social-physics systems which individuals usually compete with other travelers for position, space and velocity to minimize effort and time required to reach a specific destination. Every traveler's selfish choice leads to collectively irrational outcomes such as congestion, accidents, harmful emissions and resource depletion due to the production of travel negative externalities. It's a typical social dilemma problem which could be resolved through cooperation (Kollock 1998). A fundamental transportation theory illustrated competitive and cooperative travel behaviors as user optimal (UE) and system optimal equilibrium (SO), UE pattern results from travelers minimizing their marginal costs while SO pattern results from travelers minimizing their average costs (Wardrop 1952). SO is the results which travelers carry out cooperative actions but it won't achieve spontaneously since at SO some travelers can switch routes and lower their costs at the expense of increasing overall system costs. There are two strategies to facilitate and maintain SO pattern, the one is coercion by some authoritative transportation management departments, for instance, congestion pricing, restriction policies of motor vehicle driving or car purchasing, ect. which in fact is a kind Pigovian tax applied in transportation domain(Pigou 1920), the other is make people voluntarily make pro-social travel choice which involve how to make people adopt cooperative decision.

There are so many work studying cooperation issues in different discipline which involves economics, political science, sociology, psychology, behavior science, biology and ecology (Aral and Walker 2012; Nowak and Sigmund 2004; Nowak 2006a, 2006b; Nowak and Highfield 2012; Sugihara and Ye 2009), etc., but it's just startup in traffic transportation domain (Miller 2013), there are three basic problems are leaved to be investigated in current literature. First is what's the underling mechanism of cooperation formation and evolution in various travel decision and traffic behaviors? Second is what are the substantial forms of cooperative traffic and transportation in reality circumstances? Third is how to facilitate cooperation on all scales of transportation systems? In what follow,the three mentioned questions will be disucssed respectivelly.

\section{THE UNDERLYING MECHANISM OF COOPERATION EMERGING AND EVOLUTION}

There are intricate interactions among individuals, groups, organizations and external circumstances in traffic travel behavior of real world. Before start to discuss underlying mechanism of cooperation, the differences between various decisions and behaviors in traffic and transportation domains should be distinguished. These decisions and behaviors could be divided into two categories, the one is interactive 
decision contexts and the other is non-interactive decision contexts. For the interactive decision contexts, the work from Nowak and Sigmund (2004), Nowak (2006a, 2006b), and Ostrom (2008) are synthesized and incorporated to describe the basic principle of emerging and evolution of cooperation in person's decision. The six fundamental principles involving in formation and evolution of cooperation are reciprocity, reputation, trust, kin selection, population structure, and multi-level selection. Here we name it as general cooperative mechanism framework with interactive decision contexts (GCMF-interactive con.).

In non-interactive decision contexts, individual users make judgment and choice from all kind complicated situations combining with his information, knowledge, experiences, preference and habits, etc. If the outputs of decision behavior are benefit for the overall interests of systems which the decision maker belongs to, then the decision type is cooperative otherwise it's non-cooperative. In traffic and transportation context, for example, if individuals or group's traffic related decision is facilitate the formation of SO status, then the decision is cooperative, whereas if individuals or group's traffic related decision is conducive to generation of UE status, then the decision is non-cooperative. In non-interactive decision situation, the elements which influence the decision utility could be classified into two basic classes: endogenous elements of decision makers, and exogenous elements of decision makers. The former is consists of rational factors and irrational factors. Rational factors include all kinds of problem-solving ability, experiences, professional knowledge and skills components, etc., which are relevant rational cognitive, reasoning and logic intelligence. Irrational factors include all kinds of emotion, mood, psychological feelings, mental state and subjective preferences components, etc. The latter are more complicated and can be classified three types including physical factors (all tangible material resources), culture and Institutional factors and information resources. This process is taken regarded as general cooperative mechanism framework with non-interactive decision contexts (GCMF-non-interactive con.).

A unified general cooperative mechanism framework (GCMF) could be proposed by consolidating these two disparate structures. The general cooperative mechanism framework (GCMF) can be summarized as GCMF = GCMF-interactive context + GCMF-non-interactive context, which is demonstrated in Figure 1.

\section{THE SUBSTANTIAL MEANING OF COOPERATION AND CONCRETE FORMS OF COOPERATIVE TRAFFIC AND TRANSPORTATION SYSTEMS}

A cooperative traffic and transportation system means these three factors, effectiveness and efficiency, social equity and inclusiveness and, sustainability, are developed comprehensively and consistently. Every three factors include more elements, for instance, the effectiveness and efficiency includes at least accessibility, mobility, flexibility, safety, comfortableness, responsiveness and SO nor UE traffic network statues. The concrete forms of cooperative traffic and transportation systems can be exploited in three disparate stakeholders involving in individual travelers, traffic practitioners of academia or industry sectors, transportation relevant government administrative institutions.

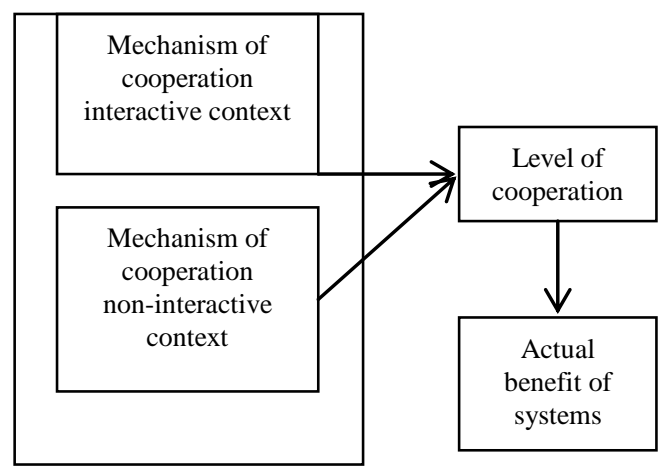

Figure 1. Schematic of unified general cooperative mechanism framework

From the points of view of individual travelers, the cooperative behavior can manifest in these below domains:

(1) Purchasing and usage decisions and behaviors of traffic traveling devices

(2) Mode choices of temporary or day-by-day travel

(3) Leveraging mobile internet or intelligent terminals to alleviate the information asymmetry status between traffic supply and demand sides.

Second, for numerous practitioners of academia or industry sectors in traffic and transportation domain, the main characteristics of cooperative traffic and transportation systems are engaged in but not limited in:

(1) Provide convenient and comprehensive traffic and travel products and services by technologies and engineering innovations.

(2) Facilitating cooperation formation on all spatiotemporal scales by combining academic insights and innovative incentives.

(3) Employing crowding to resolve practical traffic and transportation questions

Third, for government administrative institutions, the indicators of cooperative traffic and transportation systems include UO, equity, inclusiveness and sustainability which are taken as a series of main objectives for government officers concerning, so designing and implementing such systems are endowed great attentions. Typical characteristics are as follows:

(1) Explore and exploit the potentials of existing traffic resources, improve allocation and utilization efficiency of current traffic resources.

(2) Developing and deploying more effectively and diversity traffic infrastructure to satisfying diversity and volatile demands.

(3) Proposing and executing reasonable and appropriate institutional regulations and policies for cultivating more effectively, equitable and sustainable traffic and transportation systems. 


\section{HOW TO FACILITATE COOPERATION FORMATION MERGING INFORMATION RESOURCES}

It's no doubt that abundant, diverse and precise information will help actual decision and actions more effectively. Data and information are a kind of precious resources and it being in an era of information economy, especially the emerging of big data brings promising direction for creating and developing cooperative traffic and transportation systems (Cottrill and Derrible 2015). Yet the holistic performance of the traffic and transportation systems is damaged increasingly due to cumulative negative externalities of numerous selfish travel activities. How to reduce the adverse influences of cumulative negative externalities are key issues to improve the holistic performance.

Traditional solutions are internalizing the externalities by employing various institutional, managerial and technological means. There are at least three popular outstanding methods reported in existing literature. The first is based on Pigouivain theory (Pigou 1920) which realized internalizing the externalities by quota (for instance, car purchasing lottery policies in some cities of China), administrative subsidies (for instance, if customer buying private vehicles not exceeding 1.6 liter displacement he will pay lower purchasing tax) or levy taxes (for instance, ETC tolling for some specific roads or bridges carriage in China). The second is based Coase theorem (Coase 1960) which argued that an ideal status of resources configurations could be achieved by market transactions and voluntary negotiation manner. While its presumptions are so rigid that they are very difficult to satisfy in real situations especially for the reasons of traffic congestion, pollutions and some other harmful effects are a kind of the Tragedy of the Commons (Hardin 1968). The third is according social involvement thinking which proposed by Pentland (2014) who appeal to influence power of neighbors based on sociophysics and its basic notion is facilitating cooperation by a kind indirect manner that motivates his companions which will amplify the decision makers own pressures. Nevertheless these three approaches are not considering the special conducting effects of information resources primarily and firstly.

In the era of information economy, the importance and ubiquitous dissemination of all kinds signal, data and information are key factors for us to transform non-cooperative to cooperative decision and behavior in traffic and transportation systems. A conceptual framework which is named public information pools (PIP) is proposed to realize more optimal results with less expense under information influence functions. The PIP is an open sharing platform which various traffic or transportation relevant data or information can be retrieved freely for helping decision making and judgment. Decision makers employ actions reducing negative externalities positively according PIP. For example, more and more individual travelers choose Uber carpooling as a preferred travel mode which decreased the amount of private vehicles usage in many cities remarkably. Another impressed example is many individuals choose network booking travel mode as a substitution of other traditional travel mode involving in by private vehicles, by bus, by metro or by conventional taxi in China recently for the surprisingly progress on online social networks (OSN) and smart mobile applications around the world. Here the proposed PIP framework is neither administrative compulsive like Pigou taxes nor voluntary negotiation advocated in Coase theorem, whereas it presents a technical framework to eliminate information asymmetry under not knowing the actual transaction expenses thoroughly, which can reduce the transaction expenses and motivate the transformation from Pigou method to Coase theorem illustrated as in Figure 2.

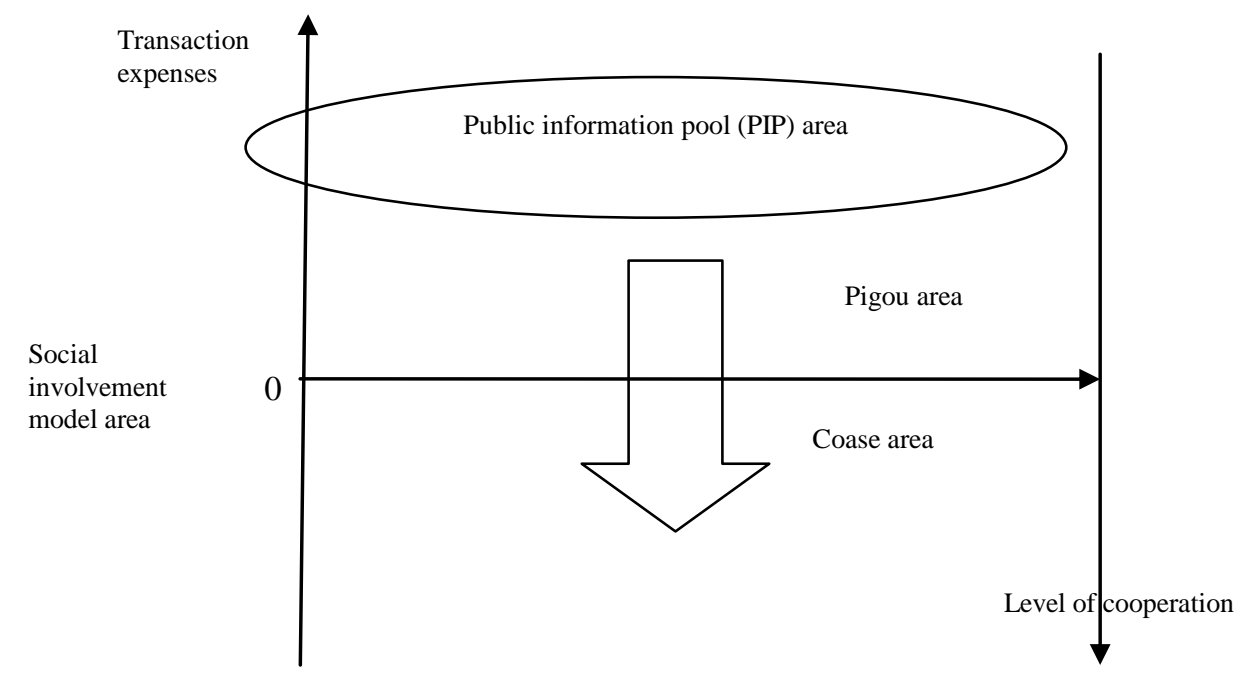

Figure 2. Comparsion of of some kinds cooperation framework 


\section{DISCUSSION AND CONCLUSION}

In this paper, some relevant topics are investigated about the cooperative issues of travel behavior and transportation systems holistically. To make sense of the intrinsic influence factors of cooperative decisions and actions, the underlying mechanism of cooperation emerging and evolution is explored. a unified general cooperative mechanism framework (GCMF) is proposed by consolidating two disparate decision contexts structures. A brief summary of some representative forms emerging in traffic and transportation practice is given. After a comprehensive analysis about facilitating the formation and development of cooperation in traffic and transports systems, a conceptual framework which is named public information pools (PIP) is proposed to generate more favorable results with less expense under information effects consideration.

The future direction may be remained in two facets, one is theoretical perspective and the other is empirical consideration. For the theoretical perspective, exploring more accurate evolutionary dynamics of cooperation in various decision contexts will require the combination of game theory, decision making theory and experimental economics methods. For the empirical consideration, leveraging incredible usage values of big data to compare and analyze various kinds of cooperative phenomena emerging in traffic and transportation fields in different communities, organizations or regions. This endeavor may lead to more meaningful and valuable insights for facilitating and cultivating a more open, efficient, fair, inclusive and sustainable traffic and transportation systems.

\section{ACKNOWLEDGEMENT}

This research was financially supported by the Natural Science Foundation of China [Grant no. 71471084]; the Key Research Funding for Wuhan Development Studies [Grant no. jhdxwyy20151204, IWHS2016104] from Wuhan Research Institute,
Jianghan University; and the International Youth Professor Project of NSU, Institutions of Higher Education, Korean Ministry of Education (Grant No. 2016-RE00648)

\section{REFERENCES}

[1] S. Aral, D. Walker, Identifying Influential and Susceptible Members of Social Networks, Science.337(2012), 337-341.

[2] W.R. Black, Sustainable transportation: a US perspective, Journal of Transport Geography . 4(1996), 151-150.

[3] R.H. Coase, The Problem of Social Cost, The Journal of Law \& Economics, 3(1960), 1-44.

[4] C.D. Cottrill , S. Derrible , Leveraging Big Data for the Development of Transport Sustainability Indicators, Journal of Urban Technology, 22 (2015), 45-64.

[5] G. Hardin, The tragedy of the commons, Science, 162 (1968), 1243-1248

[6] P. Kollock, Social Dilemmas: The Anatomy of Cooperation, Annual Review of Sociology, 24(1998), 183-214.

[7] H.J. Miller, Beyond sharing: cultivating cooperative transportation systems through geographic information science, Journal of Transport Geography, 31(2013), 296-308.

[8] M.A. Nowak, Evolutionary Dynamcis: Exploring the equations of Life, Harvard University Press, Cambridge, 2006.

[9] M.A. Nowak, Five rules for the evolution of cooperation', Science, 314(2006), 1560-1563.

[10] M.A. Nowak, R. Highfield, Super Cooperators: Altruism, Evolution, and Why We Need Each Other to Succeed, Free Press, New York, 2012.

[11] M.A. Nowak, K. Sigmund, Evolutionary dynamics of biological games, Science, 303, 793-799, 2004.

[12] E. Ostrom, Building Trust to Solve Commons Dilemmas: Taking Small Steps to Test an Evolving Theory of Collective Action, in:Levin, S.A. (Eds.) Games, Groups, and the Global . Springer, New York, Part 3, 2008, pp. 207-228.

[13] A. Pentland, Social Physics: How Good Ideas Spread-The Lessons from a New Science, Penguin Press, New York. 2014.

[14] A. C. Pigou, The Economics of Welfare, Macmillan, London, 1920.

[15] G. Sugihara, H. Ye, Complex systems: Cooperative network dynamics, Nature, 458 (2009), 979-980.

[16] J.G. Wardrop, Some theoretical aspects of road traffic research. Proceedings of the Institution of Civil Engineers, Part 1, 1(1952), 325-362. 